\title{
Centralized vs. Distributed Approaches for Encompassing Physical Impairments in Transparent Optical Networks
}

\author{
P. Castoldi ${ }^{1}$, F. Cugini ${ }^{2}$, L. Valcarenghi ${ }^{1}$, N. Sambo ${ }^{1}$, E. Le Rouzic ${ }^{3}$, M. J. Poirrier ${ }^{3}$, \\ N. Andriolli ${ }^{1}$, F. Paolucci $^{1}$, A. Giorgetti ${ }^{1}$ \\ ${ }^{1}$ Scuola Superiore Sant'Anna, Pisa, Italy, e-mail: castoldi@sssup.it \\ ${ }^{2}$ Cnit, Pisa, Italy \\ ${ }^{3}$ France Telecom, Lannion, France
}

Invited paper

\begin{abstract}
Transparent optical mesh networks are an appealing solution to provide cost-effective high bandwidth connections eliminating the need of expensive intermediate electronic regenerators. However, the implementation of transparent optical networks requires to take into account physical impairment information for effective lightpath set-up. In this paper, we present two distributed solutions to encompass physical impairments based on enhancements of the GMPLS protocol suite. Specifically, both GMPLS routing protocol and signaling protocol extensions are presented and discussed. An alternative centralized approach based on an impairment-aware Path Computation Element (PCE) is also proposed.

The distributed routing approach exhibits convergence limitations, while the distributed signaling approach is scalable and effective. The latter is then compared against the centralized PCE approach through simulations considering both a metro network and a more complex WDM network scenario. In addition, experimental implementations of the two approaches are presented. Results show the trade-off of the two approaches, demonstrating the general good performance in terms of lightpath set up time for both approaches.
\end{abstract}

Keywords: GMPLS, physical impairment, transparency, signaling protocol, Path Computation Element (PCE).

\section{Introduction}

Currently, the end-to-end lightpath provisioning over transparent optical mesh networks (i.e., networks without electronic regeneration at intermediate nodes) based on Generalized MultiProtocol Label Switching (GMPLS) protocols assumes that every route, eligible by the routing protocol, is characterized by a satisfactory signal quality. Indeed the GMPLS protocol suite does not include any information related to physical impairments [1]. Thus transparent optical network planning is based on worst case scenario, i.e. longest paths, and only limited size transparent networks are practically achievable. 
A possible approach for the enhancement of the GMPLS protocol suite to encompass physical impairment parameters has been first proposed in [2]. This approach, namely Routing Approach (RA), is based on the extension of the routing protocol, e.g. the Open Shortest Path First with GMPLS extensions (OSPF-TE). RA requires additional extensions to the routing protocol to flood physical impairment parameters. In addition RA requires, besides the presence of a Traffic Engineering Database (TED) to store bandwidth information, the availability of an additional database in every network node, namely the Physical Parameter Database (PPD). The purpose of PPD is to maintain up-to-date information on physical parameters concerning each link of the transparent network. Local information (i.e., physical parameters of the local node and of the attached links) is included in the PPD resorting to automatic monitoring and/or management systems, while remote physical parameters are obtained by exploiting the routing protocol flooding. In this way, when a connection request arrives, the Constraint Shortest Path first (CSPF) algorithm, resorting to both TED and PPD, is able to compute a route satisfying both bandwidth and optical signal quality constraints [3]. The main advantage of RA is that it is fully distributed and only minor changes are needed to the current routing protocol version. However RA suffers from several potential drawbacks. For example RA is not capable of efficiently considering intra-node physical information such as node internal losses or cross-talks and the presence of shared regenerators. In addition, it may heavily suffer from PPD inconsistency, scalability and convergence problems particularly in case of frequent link parameter changes [4] or upon failure occurrence. Yet, if only few paths in a large network have to be avoided because of their high physical impairments, each node should store and manage a large amount of information coming from the whole transparent network. As a consequence, to avoid few impaired routes (e.g., longest paths), the routing protocol must continuously disseminate all the relevant parameters. Moreover, a multi-constrained path computation is required for achieving both optimal network performance and sufficient quality of the optical signal. This may heavily impact the load of the node's processing unit (CPU), determining large computation time and delaying the lightpath establishment. For these reasons, RA gives minor advantages as compared to the introduced complexity and alternative approaches have then been proposed.

In this paper, two different approaches alternative to RA are considered and evaluated.

The first considered approach, hereafter addressed as Signaling Approach (SA), has been proposed in [5] and elaborated more in [6]. SA is based on the enhancement of the signaling protocol, e.g. Resource Reservation Protocol with GMPLS extensions (RSVP-TE), to encompass physical impairments during lightpath establishment.

The second considered approach, hereafter addressed as Path Computation Element (PCE, [7]) based approach (PCE-A), has been first proposed in [8]. PCE-A is based on the computation of lightpath physical impairments performed in a centralized way by the PCE.

In this paper, for the first time, the SA and PCE-A performance are evaluated through simulations on realistic Metro network and WDM network scenarios considering an accurate physical impairment modeling. Moreover, the experimental implementations of the two different approaches are presented. 


\section{Signaling Approach}

The Signaling Approach (SA) is based on the dynamic estimation of the optical signal quality during the signaling phase of the lightpath set up process. No modifications are introduced in the OSPF-TE routing protocol which elaborates routes ignoring physical impairments. The RSVP-TE signaling protocol is extended to collect the physical parameter values characterizing every traversed node (e.g., Photonic Cross-Connect (PXC)) from the source to the destination node. The source node generates an RSVP Path message extended with a novel Cumulated Physical Parameter (CPP) object, which contains the array of physical parameters of the transmitting interface and of its outgoing link. Every traversed node, before propagating the message, updates the CPP object by adding its own local parameter values (i.e., intra-node and outgoing link parameters). Admission control at the destination node compares the overall accumulated parameters with the required parameter values that characterize its receiver interface. If the accumulated parameters are within an acceptable range, the lightpath set up request is accepted and an RSVP Resv message is sent back to the source node. Otherwise the lightpath request is rejected and an RSVP Error is sent back to the source node. If the request is rejected, further set up attempts following possibly link-disjoint routes are triggered. However, the successive set up attempts may also fail, excessively delaying the lightpath establishment process.

To overcome these issues, a novel RSVP object called Physical Parameter (PP) object and a locally managed Physical Parameter Database (PPD) are introduced as optional extensions. PP object contains the array of physical parameter information that characterizes every traversed link and that contributes to the CPP object. PPD stores the physical parameters of all network links, retrieved resorting to every transit signaling message carrying PP objects. PPD is used to provide a prediction of the cumulated physical parameters along the computed path. If the predicted value is outside the acceptable range, then the computed path is not validated and a different path is considered. On the contrary, if the predicted value is within the acceptable range, the source node starts the signaling phase. PPD might be not up-to-date, so the set up attempt along validated paths might be rejected due to unacceptable physical impairments. In this case an alternative path, possibly link-disjoint, is then computed and evaluated. Proper expiration time of PPD parameters is also considered.

\section{PCE-based Approach}

Path Computation Element (PCE) is defined in [7] as an entity (component, application, or network node) that is capable of computing a network path or route based on a network graph and applying computational constraints during the computation. PCE has been introduced mainly to perform complex route computation on behalf of network nodes in particular in the cases of partial visibility of the network topology to the destination, i.e. multi-area, multi-domain and multi-layer networks. PCE typically takes into account bandwidth requirements, QoS parameters and survivability aspects. 
The considered PCE-based Approach (PCE-A) resorts to the PCE utilization also in the case of transparent optical networks. For this purpose, the PCE has to be aware of additional information referring to the physical parameters. The complete set of physical parameters is stored by the PCE in a locally managed Physical Parameter Database (PPD) equal to the one used by SA. In this case, however, all the PPD parameters are obtained by the Management System or through a performance monitoring system. In this approach, no modifications are introduced into the Control Plane, thus neither the routing nor the signaling protocols are enhanced with further extensions. Whenever the PCE receives a path computation request from a Path Computation Client (PCC), it computes the required path taking into account the TE information and the physical impairment constraints. Then, the computed route is converted into an explicit route (i.e., a set of nodes and links) and returned to the PCC for establishing the lightpath by means of a distributed signaling protocol.

\section{Network scenario and simulation results}

The performance of SA and PCE-A approaches has been evaluated by means of a $\mathrm{C}++$ event-driven network simulator considering two different network scenarios.

The first scenario aims at reproducing the behavior of a transparent Metro network made of just passive devices (e.g., PXC with no in-line optical amplification) between the source and destination transponders (e.g., optical Ethernet transponders. Fig. 1 shows the considered topology of 6 PXCs and 8 links. Network links are characterized by a physical parameter $l_{i j}$ carried by the CPP object representing the link length between PXC $i$ and $j$. Fig. 1 also reports the $l_{i j}$ values in kilometers for the reference Metro network. The impairments introduced by every transit PXC $i$ are also taken into account adding to CPP an equivalent distance $l_{i}$ [2] equal to $3 \mathrm{~km}$. A maximum acceptable cumulated value $L_{M A X}=100 \mathrm{~km}$ is imposed.

The second scenario aims at reproducing the behavior of a transparent WDM backbone network. Fig. 2 shows the considered Pan-European topology with 32 links and 17 PXCs equipped with WDM transponders at $10 \mathrm{Gbps}$. In order to determine the optical signal quality, a mixed Q factor and Bit Error Rate (BER) criterion [9] with a direct relation to Optical Signal to Noise Ratio (OSNR) [10] is considered. OSNR value represents the optical signal quality and modeled physical impairments are expressed as penalties [9] on OSNR value. Beside noise, modeled impairments are: PMD (Polarization Mode Dispersion), CD (Chromatic Dispersion) and self-phase modulation (SPM) using the non linear phase shift parameter $\phi_{\mathrm{NL}}$. All modeled effects are represented by four parameters that cumulate linearly: $1 / \mathrm{OSNR}_{\mathrm{S}} \mathrm{PM}^{2}, \mathrm{CD}$ and $\phi_{\mathrm{NL}}$. The four parameters are included in the PP and CPP objects to describe the impairments associated to links and paths respectively. The final OSNR value at the destination node is obtained from the cumulated OSNR value by considering the penalties induced by the other parameters [9]. The path is rejected if the final OSNR is below a threshold equal to $13 \mathrm{~dB}$ (this value includes also the margins for the non modeled impairments such as Polarization Dependant Loss, filtering, crosstalk, system ageing and other non linear effects). The path is also rejected if one parameter 
exceeds the model validity domain (e.g., $1 \mathrm{rad}$ for $\phi_{\mathrm{NL}}, 4 \mathrm{~dB}$ for penalty due to PMD or $\mathrm{CD}$ ).

In both scenarios lightpath requests are dynamically generated with uniform distribution among all node pairs. Network load is kept limited in order to have lightpath blocking due exclusively to unacceptable optical signal quality.

The performance of SA is shown in Figs. 3-5. The curves show the percentage of established lightpaths within $n$ set up attempts as a function of the generated unidirectional lightpath requests. The percentage of established lightpaths is computed for a 100 requests observation window and averaged over 100 repetitions. Fig. 3 refers to the Metro network scenario where just the equivalent length is considered. Fig. 4 refers to the WDM Pan-European network where the more accurate model is needed. Results show that in both Metro and WDM backbone network scenarios SA achieves constant performance above $96 \%$ of established lightpaths at the first set up attempt. Moreover Figs. 3 and 4 show that all the requests are successfully established within the second attempt in the Metro network and within the third attempt in the WDM backbone network. Fig. 5 shows the performance of SA in the WDM backbone network when also the optional PP extensions are utilized. Results show that the percentage of established lightpaths increases with the amount of generated requests up to $99 \%$ at the first set up attempt and $100 \%$ already at the second set up attempt, thus guaranteeing a limited amount of signaling messages and set up delay.

In PCE-A, PCE is aware of all network physical parameters. PCE then performs effective multi-constraint route computation [3] thus guaranteeing the establishment of all lightpaths at the first signaling attempt (if the PPD information is up-to-date).

\section{Experimental evaluation: Signaling Approach (SA)}

The testbed, in which the SA is implemented, is shown in Fig. 6. It reproduces a portion of a typical Metro network where two edge routers (A and B) are connected through a transparent network of four PXCs. Edge routers are equipped with optical Ethernet interfaces (1000BaseLX) whose maximum span reach is equal to $10 \mathrm{~km}$. In this testbed again we assume the maximum distance as the only relevant physical parameter. PXC1 and PXC2 serve router A and B, respectively, to dynamically set up optical connections within the domain of transparency. Every link between PXCs is 3 $\mathrm{km}$ long except for PXC3-PXC2 link which is $6 \mathrm{~km}$ long. Linux Boxes (LB) are employed to manage PXCs via the parallel port. RSVP-TE messages extended with the previously described objects are exchanged among the LBs on the out-of-band Ethernet control plane. The proposed approach allows also to take into account intranode information without causing, because of the absence of flooding, scalability problems (e.g., for PXC1 we estimate an equivalent distance of $1 \mathrm{~km}$ between port 1 and 2, $1.5 \mathrm{~km}$ between port 1 and 3, etc.). Every node also maintains a local PPD database containing the physical information, i.e. length, describing the attached links, e.g. LB3 maintains information for the internal PXC3 physical parameters and for links PXC1-PXC3 and PXC3-PXC2. 
A connection request is generated at source router $\mathrm{A}$ to destination router B and it is handled by PXC1. Because the routing algorithm is unaware of physical impairments, the two possible paths are considered perfectly identical. As a worst case scenario, the route passing through PXC3 is selected. A first exchange of modified RSVP messages allows LB1 to know that excessive physical impairments do not allow the lightpath establishment and a second successful set up attempt is performed. The complete message exchange is presented in Fig. 7, showing that the overall RSVP packet exchange takes less than 40 milliseconds.

\section{Experimental evaluation: PCE-based Approach (PCE-A)}

The PCE architecture with the implemented activation modules is depicted in Fig. 8. The Label Switched Path (LSP) Request Collector (LSP-RC) module accepts both single and multiple LSP requests. LSP-RC produces an LSP XML file which contains, for every LSP request, the Ingress and Egress nodes, the bandwidth and the physical impairment requirements in case of transparent networks. The Traffic Engineering Database (TED) module collects information directly from the TED database stored in network nodes and maintained by the OSPF-TE protocol. The TED module downloads the complete TED from the node in the form of an XML file by connecting to the node proprietary User-to-Network Interface (UNI) through a TCP socket. TED download is triggered by the LSP-RC module upon a new LSP Request. The TED module requires a bidirectional communication between the PCE and the node management interface per routing Area. Indeed, TE information (i.e., Opaque Link State Advertisement (LSA)) is flooded only within the Area scope. EXtensible Stylesheet Language Transformation (XSLT) is then applied to the downloaded XML files in order to combine the information coming from different routing Areas and to prune redundant and useless information. XSLT elaboration produces a single TED XML file describing the network topology and the available bandwidth for every link of the network.

The Physical Parameter Database (PPD) maintains up-to-date information on physical parameters concerning any network link. Without loss of generality, we consider just the link length to model all the relevant physical impairments. An Optical Time Domain Reflectometer (OTDR) is used to populate the PPD database. One PPD XML file is then generated describing the physical parameter values of every network link. The three XML files obtained from the three aforementioned modules are then elaborated to compute all the required strict routes. In particular, the three files are first elaborated by a PCE Interface (PCE-I) module implemented in C code. The PCE-I generates an LP formulation of the lightpath provisioning problem and passes it to the PCE which is based on a Linear Programming (LP) solver. The implemented objective function minimizes the maximum link bandwidth utilization (least-fill policy). The output file generated by the PCE contains the strict routes of every LSP request which satisfy the required constraint in terms of bandwidth and optical signal quality. A further elaboration is performed by the Configuration File Builder (CFB) module to produce a set of XML files containing the computed routes formatted so that they can be directly uploaded as node configurations. One XML 
Configuration file per Ingress node is generated. An LSP Activator (LSP-A) module, which exploits the node proprietary UNI, is then utilized to contemporarily upload and activate the proper XML Configuration File in each involved Ingress node.

The PCE-A implementation is applied to the general Metro scenario shown in Fig. 6. In this case, the PCE is able to select the feasible route traversing PXC3. The overall delay introduced by the PCE-A has been measured in only $0.320 \mathrm{~s}$.

\section{Discussion and conclusion}

In this study two different approaches to encompass physical impairments in the Control Plane of transparent optical networks are evaluated. The Signaling-based (SA) and the PCE-based (PCE-A) approaches are analyzed through numerical simulations and experimental implementations.

Results show that under realistic Metro and WDM backbone network scenarios the two approaches perform well. SA is fast, requiring very few set up attempts before completing the lightpath establishment. Moreover it is fully distributed and it is potentially more effective in taking into account sudden physical parameter changes. However, it requires Control Plane extensions and it can provide less effective Traffic Engineering (TE) solutions. PCE-A guarantees effective TE solutions and it can also exploit ad-hoc routing strategies. Moreover, PCE-A does not require additional Control Plane extensions. However, it may suffer from scalability problems and may rely on outdated information.

Acknowledgments. This work has been partially supported by NoE e-Photon/One+ and by IST FP6 NOBEL Phase 2 project.

\section{References}

1. R. Martínez, C. Pinart, F. Cugini, N. Andriolli, L. Valcarenghi, P. Castoldi, L. Wosinska, "Challenges and requirements for introducing impairment-awareness into the management and control planes of ASON/GMPLS WDM networks", IEEE Comm. Mag., Dec. 06.

2. J. Strand, et al., "Issues for routing in the optical layer", IEEE Comm. Mag., Feb. 2001.

3. P. Kulkarni, A. Tzanakaki, C. M. Machuka, I. Tomkos, "Benefits of Q-factor based routing in WDM metro networks", ECOC 2005.

4. R. Chraplyvy, "Equalization in Amplified WDM Transmission Systems", PTL Aug 92.

5. F. Cugini, N. Andriolli, L. Valcarenghi, P. Castoldi, "Physical impairment aware signaling for dynamic lightpath set up" ECOC 2005.

6. N. Sambo, A. Giorgetti, N. Andriolli, F. Cugini, L. Valcarenghi, P. Castoldi, "GMPLS Signaling Feedback for Encompassing Physical Impairments in Transparent Optical Networks", GLOBECOM 2006.

7. A. Farrel, "A Path Computation Element (PCE)-Based Architecture”, RFC 4655, Aug. 06.

8. F. Cugini, F. Paolucci, L. Valcarenghi, P. Castoldi, "Implementing a Path Computation Element (PCE) to encompass physical impairments in transparent networks", OFC 2007.

9. I. Kaminov, T. Li, "Optical Fiber Telecommunications", vol. IV, Elsevier, 2002.

10. Huang Y. et al., "Connection Provisioning with Transmission Impairment Consideration in Optical WDM Networks with High-Speed Channels", JLT Vol. 23, No. 3, Mar. 2005. 

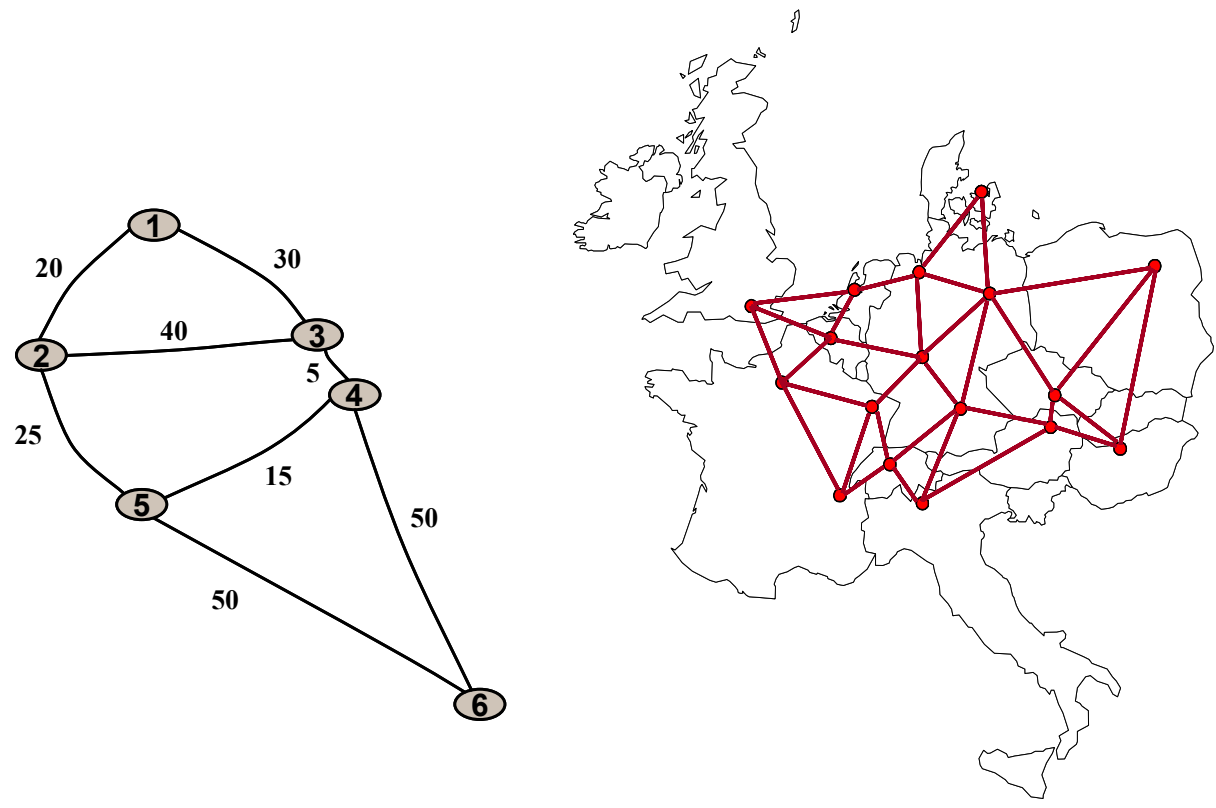

Fig. 1: Transparent Metro network

Fig. 2: WDM backbone network

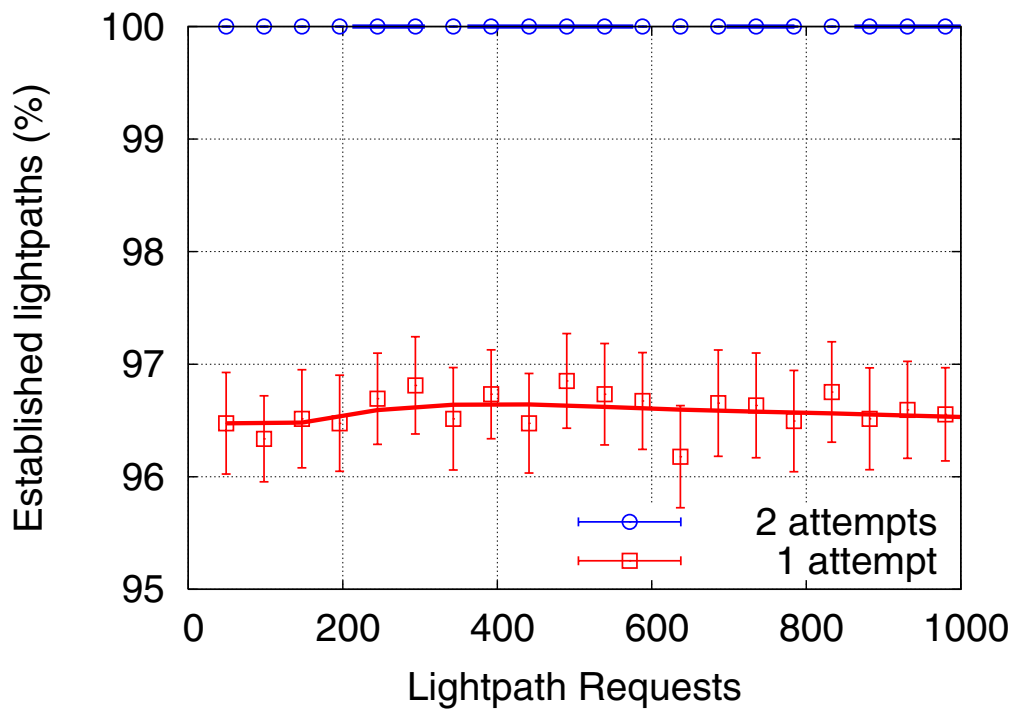

Fig. 3: Signaling Approach (SA), percentage of established lightpath (Metro network) 


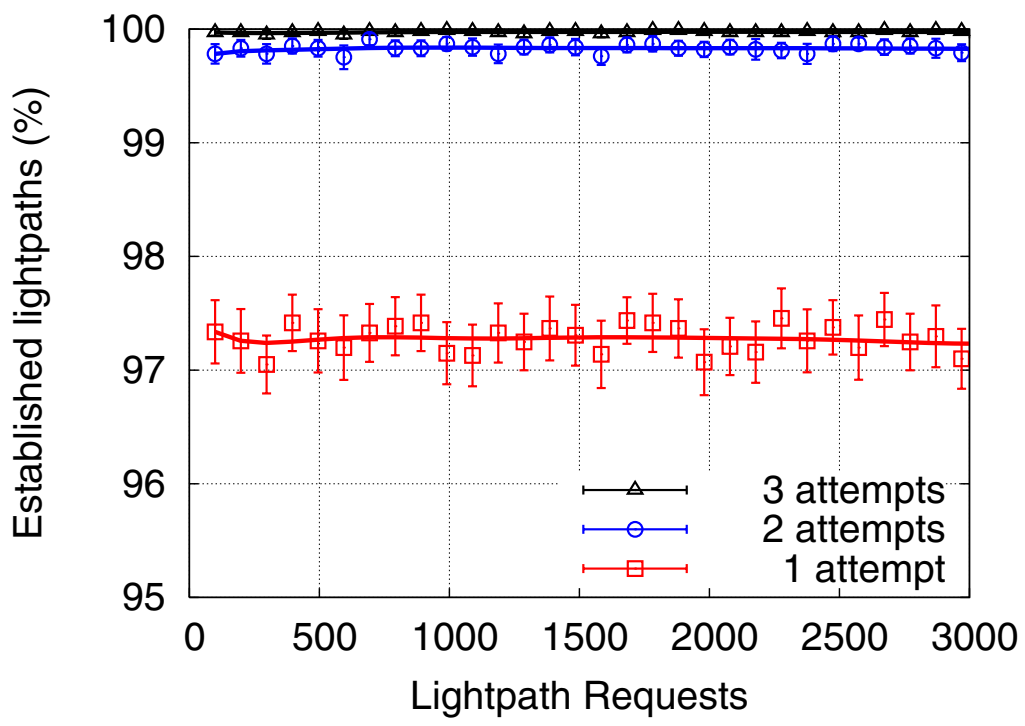

Fig. 4: Signaling Approach (SA), percentage of established lightpath (WDM backbone network).

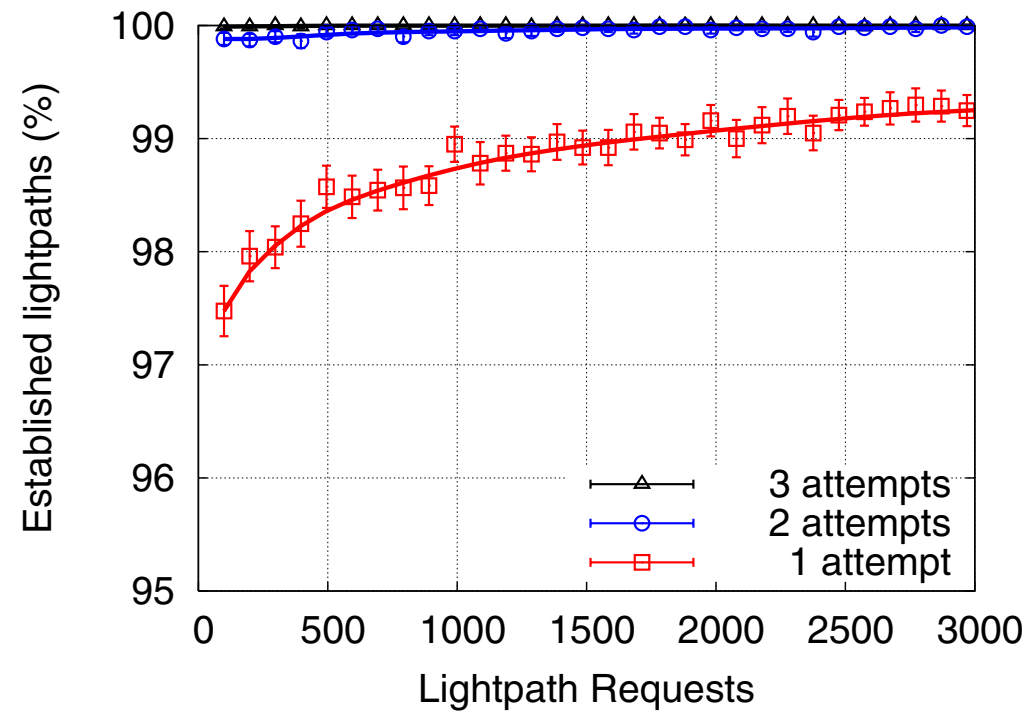

Fig. 5: Signaling Approach (SA), percentage of established lightpath resorting to PPD (WDM backbone network). 


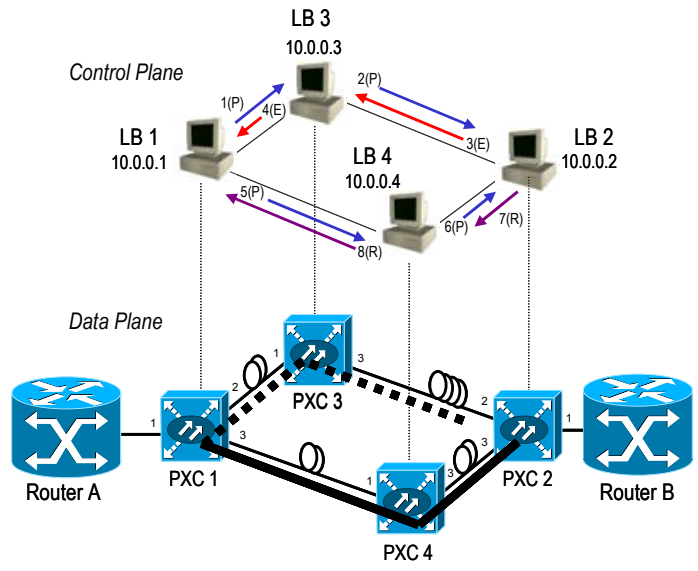

Fig. 6: Experimental implementation

\begin{tabular}{|r|l|l|l|l|l|}
\hline No. . & Time & Source & Destination & Protocol & Info \\
\hline 3 & 2.876711 & 10.0 .0 .1 & 10.0 .0 .3 & RSVP & PATH Message. SESSION: IPV4 \\
\hline 4 & 2.883147 & 10.0 .0 .3 & 10.0 .0 .2 & RSVP & PATH Message. SESSION: IPV4, \\
5 & 2.889287 & 10.0 .0 .2 & 10.0 .0 .3 & RSVP & PATH ERROR Message. SESSION: \\
6 & 2.892644 & 10.0 .0 .3 & 10.0 .0 .1 & RSVP & PATH ERROR Message. SESSION: \\
7 & 2.898569 & 10.0 .0 .1 & 10.0 .0 .4 & RSVP & PATH Message. SESSION: IPV4, \\
8 & 2.902485 & 10.0 .0 .4 & 10.0 .0 .2 & RSVP & PATH Message. SESSION: IPV4, \\
9 & 2.909444 & 10.0 .0 .2 & 10.0 .0 .4 & RSVP & RESV Message. SESSION: IPV4, \\
10 & 2.912848 & 10.0 .0 .4 & 10.0 .0 .1 & RSVP & RESV Message. SESSION: IPV4,
\end{tabular}

Fig. 7: GMPLS message exchange over the out-of-band control plane; time in seconds.

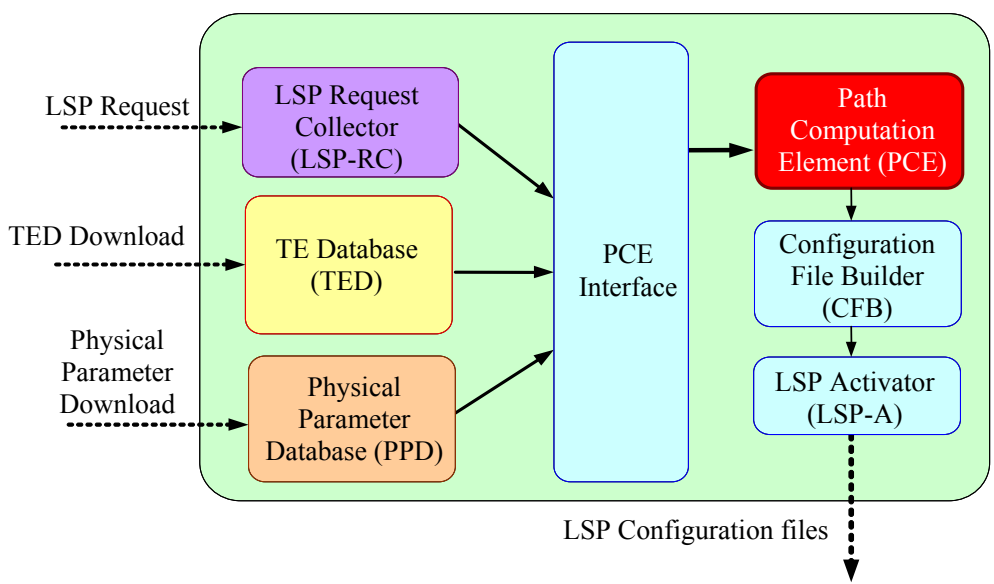

Fig. 8: PCE-based Approach implementation 